\title{
Product Design Decision Oriented by Hierarchical Relevance of User's First Perceptual Demand
}

\author{
Yi Wang*, Zipei Ding, Sichao Fan \\ Department of industrial design, Xi'an university of Technology,710054,China
}

\begin{abstract}
User's perceptual demands for products usually show "multi-dimensional, multi-level, dynamic, and ambiguous" features. Emotional words and phrases are often used by users to express their perceptual demands for products. In this paper, the dynamic intuitionistic fuzzy multi-attribute decision-making method is adopted to establish the hierarchy of demands. The correlation measure of intuitionistic fuzzy sets is used to calculate the correlation between the perceptual demand set and the semantic set with emotional expressions. Mapping the results to target users' typical characteristics, we can acquire the users' hierarchy of demands to designed products. Taking the design of a headset as the case, product perception evaluation experiments and relevant data analysis of 10 different design plans are carried out to clarify the ideal product's "position" in the semantic space of typical user group. The hierarchy of perceptual demands of users to the headset is successfully established, which proved the feasibility of this method.
\end{abstract}

\section{Introduction}

The growing update frequency of consuming goods, to some extent, has encouraged the individualized perceptual demands of consumers for products ${ }^{[1]}$. In order to meet user's diversified anticipations, an efficient and accurate data collection method or a model to analyze user's multidimensional perceptual demands have become the recent research focus ${ }^{[2]}$. What's more, some researchers tried to describe consumers' emotional demands based on onlineshop feedbacks and Dynamic Evolving Neural-Fuzzy Inference System (DENFIS), with the results, in a good way, reflecting the dynamic nature of users' preference ${ }^{[3]}$. Back Propagation Neural Network (BPNN) is used to map users' perceptual images to product parameters and to predict users' psychological assessment to products ${ }^{[4]}$. However, further studies should be carried out to explore the hierarchy of multi-dimensional perceptual demands ${ }^{[5]}$, and users'diversified but correlated demands make design even harder. Based on the problem, a dynamic intuitionistic fuzzy multi-attribute decision-making method $^{[6]}$ is proposed in this study to rank users' diversified perceptual demands, establishing the hierarchy of their demands. The correlation measure of intuitionistic fuzzy sets is used to analyze the semantic correlation of users' multi-dimensional perceptual demands. Classifying the result of sematic correlation in accordance with typical users' characteristics, we will work out a direct description of users' comprehensive and dynamic perception to design plans, ensuring the scientificity and reliability of the evaluation to product design based on users' perceptual demands.

\section{$2 \quad$ Analysis of users' perceptual demands and semantic association}

\subsection{Multi-dimensional perceptual demands of users}

With the continuous improvement of scientific and technological productivity, the design of products must meet the demands about function and emotion of users. However, User's perceptual demands for products usually show multi-dimensional and uncertain. Therefore, scholars from various countries have conducted research and analysis on users' multidimensional perceptual demands. At present, there are some models which describe users' demands based on axiomatic design, they use joint analysis to quantify the demands of users and transform them into specific product design indicators and parameters ${ }^{[7]}$. Alternatively, the user emotions are layered according to the Kano demand model, and then the product design features are classified according to the Norman model, and then a user emotional demand hierarchy and product design feature matching model is established ${ }^{[8]}$ At the same time, according to the multi-level and dynamic nature of users' demands, a multi-dimensional users' demands acquisition method for products and users is proposed. The analytic hierarchy process is applied to evaluate the established multi-level users' demands, and the analysis process and method of users' demands information are established, so as to provide designers with a clear product concept design reference direction $^{[9]}$.

\footnotetext{
"Corresponding author's e-mail: wy2005@xaut.edu.cn
} 


\subsection{Multi-dimensional perceptual demands of users with semantic association}

Although the user's perceptual demands for the products are diverse, they also show some certain level. This level of demand can be distinguished by major, minor, and weak. Although the user's semantic expression of the product is subjective and uncertain, the vocabulary of the semantic expression is still relevant. For example, in a user's perceptual demand for certain product, "sport" has a stronger association with "solidity and smooth" than with " solemn and softness ". "Sport" and " solidity " are closely related in a higher level, On the contrary, " quiet " and "sport" are antonyms, the latter is in low level relation with " solidity". Considering the multi-attributes of products and the diverse, complex dynamic (dynamics of market demand) and multi-user demands, the study proposes to extract the emotional words about perceptual demands that a user frequently selects $Y=\left\{Y_{1}, Y_{2}, \ldots Y_{n}\right\}$ from to establish the user's perceptual information set about the product. We use the dynamic intuitionistic fuzzy multiattribute decision-making method to establish the hierarchy of "demand $R_{i}$ " and select the perceptual demands of higher hierarchy as the semantic axis $M R_{j}$ in the "perceptual demand semantic space". When $\mathrm{j}=3$, the semantic axis $M R_{3}$ forms a three-dimensional inductive requirement semantic space with $M R_{2}$ and $M R_{1}$.

We can calculate the association level of the products perceptual information set to the product plan $\mathrm{Pi}$ and the relationship semantic axis through the correlation measure of the intuitionistic fuzzy set, and determine the position of the product plan in the semantic space of users' requirement, as shown in Figure1.The position of the product in threedimensional perceptual semantic space can be found.

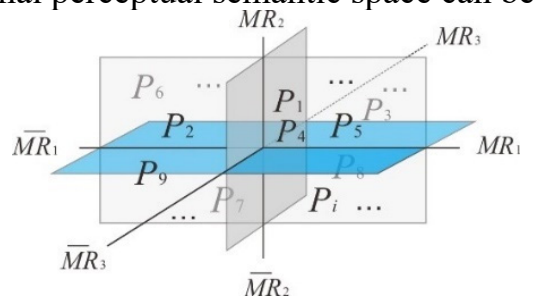

Figure 1. Location of products in the Three-dimensional semantic space.

\section{Algorithm Research}

\subsection{Multi-dimensional perceptual demands of users with semantic association}

The perceptual demands of users are fuzzy, dynamic, diverse and prioritized. In terms of the priority feature, this paper used the Dynamic Intuitionistic Fuzzy Multiattribute Decision-making method to rank the perceptual demands of users, so as to decide the prioritized and important perceptual information in their needs. Assume the perceptual demand information set of users for product is $Y=\left\{Y_{l}, Y_{2}, \ldots Y_{n}\right\} ; G=\left\{G_{l}, G_{2}, \ldots G_{m}\right\}$ is the attribute set of product, with the weight vector of each is $w=\left(w_{1}, w_{2}, \ldots, w_{m}\right)^{T}, \quad$ where $w_{j} \geq 0(j=1,2, \ldots, m), w_{j} \geq 0(j=1,2, \ldots, m), \sum_{j=1}^{m} w_{j}=1$. Since the market is dynamic, the perceptual demands of users for product are also in dynamic changes. Therefore, a time parameter $t_{k}(k=1,2, \ldots, p)$, is added to obtain $p$ different time periods of user's demand. Weight vector is given to each time period according to its influence on prediction or decision-making for the dynamic demands of product.

$\omega(t)=\left(\omega\left(t_{1}\right), \omega\left(t_{2}\right), \ldots, \omega\left(t_{p}\right)\right)^{T}, \omega\left(t_{k}\right) \geq 0(k=1,2, \ldots, P), \sum_{k=1}^{p} \omega\left(t_{k}\right)=1 ;$

$D\left(t_{k}\right)=\left(\left(d_{i j}\left(t_{k}\right)\right)_{n \times m}\right)$ is the intuitionistic fuzzy decision matrix of the time period $t_{k}$, where

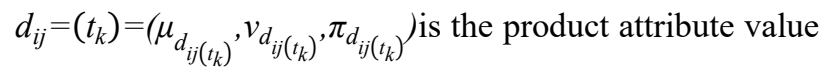
expressed by the fuzzy matrix , $\mu_{d_{i j\left(t_{k}\right)}}$ represents that the degree of solution $Y_{i}$ meets the product attribute $G_{j}$ in time period $t_{k}, v_{d_{i j}\left(t_{k}\right)}$ represents that the degree of solution $Y_{i}$ fails to meet the product attribute $G_{j}$ in time period $t_{k}$, and $\pi_{d_{i j\left(t_{k}\right)}}$ represents the degree of non-determinacy of solution $Y_{i}$ to the attribute $G_{j}$, then $\mu_{d_{i j\left(t_{k}\right)}} \in[0,1], v_{d_{i j\left(t_{k}\right)}} \in$ $[0,1], \mu_{d_{i j\left(t_{k}\right)}}+v_{d_{i j\left(t_{k}\right)}} \leq 1, \pi_{d_{i j\left(t_{k}\right)}}=1-\mu_{d_{i j\left(t_{k}\right)}}-$

$v_{d_{i j\left(t_{k}\right)}}(i=1,2, \ldots, n, j=1,2, \ldots, \mathrm{m})$, The Dynamic Intuitionistic Fuzzy Multi-attribute Decision-making method is used to decide the hierarchy of needs of users, shown as follows:

Step 1: Use the DIFWA operator:

$$
\begin{gathered}
d_{i j}=D I F W A_{\omega(t)}\left(d_{i j}\left(t_{1}\right), d_{i j}\left(t_{2}\right), \ldots, d_{i j}\left(t_{p}\right)\right) \\
=\left(1-\prod_{\mathrm{k}=1}^{p}\left(1-\mu_{d_{i j\left(t_{k}\right)}}\right)^{\omega\left(t_{k}\right)}, \prod_{\mathrm{k}=1}^{p} v_{d_{i j}\left(t_{k}\right)}^{\omega\left(t_{k}\right)}, \prod_{\mathrm{k}=1}^{p}\left(1-\mu_{d_{i j\left(t_{k}\right)}}\right)^{\omega\left(t_{k}\right)}-\right. \\
\prod_{\mathrm{k}=1}^{p} v_{d_{i j}\left(t_{k}\right)}^{\omega\left(t_{k}\right)}, \quad(i=1,2, \ldots n ; j=1,2, \ldots, m) \quad
\end{gathered}
$$

Step 2: Define the positive ideal intuitionistic fuzzy point and negative ideal intuitionistic fuzzy point respectively: $\quad Y^{+}=\left(a_{1}{ }^{+}, a_{2}{ }^{+}, \ldots, a_{m}{ }^{+},\right)^{T}$ and $Y=\left(a_{1}^{-}, a_{2}{ }^{-}, \ldots, a_{m}{ }^{-},\right)^{T}$, where $a_{i}^{+}=(1,0,0)(i=1,2, \ldots, m)$, referring to $\mathrm{m}$ maximum intuitionistic fuzzy numbers, while $a_{i}{ }^{-}=(1,0,0)(i=1,2, \ldots, m)$, representing $\mathrm{m}$ minimum intuitionistic fuzzy numbers. Solution set $Y_{i}=$ $\left(d_{i 1}, d_{i 2}, \ldots, d_{i m},\right)^{T}(i=1,2, \ldots, n)$

Step 3: Respectively calculate the distance between the positive ideal intuitionistic fuzzy point $Y^{+}=$ $\left(a_{1}{ }^{+}, a_{2}{ }^{+}, \ldots, a_{m}{ }^{+},\right)^{T}$ and the negative ideal intuitionistic fuzzy point $Y^{-}=\left(a_{1}{ }^{-}, a_{2}{ }^{-}, \ldots, a_{m}{ }^{-} \text {, }\right)^{T}$ of solution $Y_{i}$ :

$d\left(Y_{i}, Y^{+}\right)=\sum_{j=1}^{m} w_{j} d\left(d_{i j}, Y_{j}^{+}\right)=\sum_{j=1}^{m} w_{j}\left(1-\mu_{i j}\right)$

$\mathrm{d}\left(Y_{i}, Y^{-}\right)=\sum_{j=1}^{m} w_{j} d\left(d_{i j}, a_{j}^{-}\right)=\sum_{j=1}^{m} w_{j}\left(1-v_{i j}\right)$

Where $d_{i j}=\left(\mu_{i j}, v_{i j}, \pi_{i j}\right)(i=1,2, \ldots, n ; j=1,2, \ldots, m)$.

Step 4: Calculate the proximity coefficient of each solution: 
$c\left(Y_{i}\right)=\frac{d\left(Y_{i}, Y\right)}{d\left(Y_{i}, Y^{+}\right)+d\left(Y_{i}, Y\right)}(i=1,2, \ldots, n)$

According to Equations (3) and (4),

$$
\left.c\left(Y_{i}\right)=\frac{\sum_{j=1}^{m} w_{j}\left(1-v_{i j}\right)}{\sum_{j=1}^{m} w_{j}\left(1+\pi_{i j}\right)}(i=1,2, \ldots, n)\right)
$$

Step 5: Judge the proximity coefficient, rank and pick over the semantic vocabulary $Y_{i}=\left\{Y_{1}, Y_{2}, \ldots Y_{n}\right\}$ in all information sets of perceptual needs. The larger the value of $c\left(Y_{i}\right)$ is, the easier the semantic vocabulary $Y_{i}$ is perceived by users. In this study, semantic vocabularies with higher prioritization rank are selected as the prioritized and important perceptual demand information of users for product, then the information is taken as the semantic axis $M R_{j}$ or primary demand $F R$ in the multidimensional perceptual demand semantic space.

\subsection{Semantic association analysis of multi- dimensional perceptual information}

Using dynamic intuitionistic fuzzy multi-attribute decision-making to rank perceptual needs, selecting the user's priority and important perceptual requirements for the product as the two-dimensional perceptual requirements semantic spatial feature semantic axis $M R_{j}$, and the remaining perceptual requirements through the use of association measures of intuitionistic fuzzy sets ${ }^{[7]}$ After analysis, the position of these perceptual requirements in the two-dimensional requirement semantic space is obtained. This is a process of perceptual demand clustering through semantic association analysis.

If the association measure of intuitionistic fuzzy sets is used to calculate the correlation between the semantic set of product perceptual demand information and typical characteristics of target users, it can comprehensively describe the position of $\mathrm{n}$-dimensional perceptual demand information corresponding to the user's perception of product solutions in the semantic space of typical characteristics of user groups as shown in Figure 2.

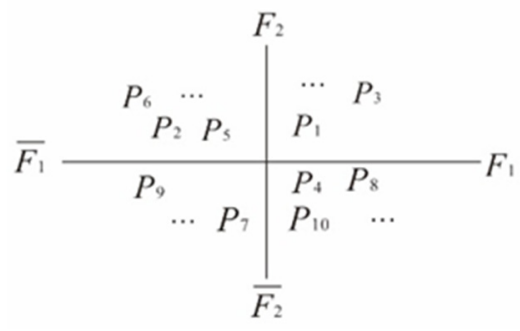

Figure 2. Location of products in the Three-dimensional semantic space.
Suppose $X=\left\{x_{1}, x_{2}, \ldots, x_{n}\right\}$ is a finite set, the intuitionistic fuzzy set are $\mathrm{A}_{1}=\left\{\left\langle x_{i}, \mu_{A_{1}}\left(x_{i}\right), v_{l}\left(x_{i}\right) \mid x_{i} \in X\right\rangle\right\}$ and $\mathrm{A}_{2}=\left\{\left\langle x_{i}, \mu_{A_{2}}\left(x_{i}\right), v_{A_{2}}\left(x_{i}\right) \mid x_{i} \in X\right\rangle\right\}$, then the correlation coefficient of the intuitionistic fuzzy set $A_{1}, A_{2}$ is:

$\rho_{1}\left(A_{1}, A_{2}\right)=\frac{1}{2 n} \sum_{i=1}^{n}\left(\frac{\Delta \mu_{\min }+\Delta \mu_{\max }}{\Delta \mu_{i}+\Delta \mu_{\max }}+\frac{\Delta v_{\min }+\Delta v_{\max }}{\Delta v_{i}+\Delta v_{\max }}\right)$

Where $\quad \Delta \mu_{i}=\left|\mu_{A_{1}}\left(x_{i}\right)-\mu_{A_{2}}\left(x_{i}\right)\right|, \Delta v_{i}=\mid v_{A_{1}}\left(x_{i}\right)$ $v_{A_{2}}\left(x_{i}\right)\left|, \Delta \mu_{\text {min }}=\min _{i}=\right| \mu_{A_{1}}\left(x_{i}\right)$ -

$\mu_{A_{2}}\left(x_{i}\right)\left|, \Delta v_{\min }=\min _{i}\right| v_{A_{1}}\left(x_{i}\right)-v_{A_{2}}\left(x_{i}\right) \mid$

$\Delta \mu_{\text {max }}=\max _{i}=\left|\mu_{A_{1}}\left(x_{i}\right)-\mu_{A_{2}}\left(x_{i}\right)\right|, \Delta v_{\max }=\max _{i} \mid v_{A_{1}}\left(x_{i}\right)-$

$v_{A_{2}}\left(x_{i}\right) \mid$.

In terms of the multi-attribute decision-making, each attribute has different importance to the product. If the weight of product element $x_{i} \in X$ is taken into account, Equation (7) can be generalized as:

$\rho_{2}\left(A_{1}, A_{2}\right)_{2} \frac{1}{2} \sum_{i=1}^{n} \omega_{i}\left(\frac{\Delta \mu_{\min }+\Delta \mu_{\max }}{\Delta \mu_{i}+\Delta \mu_{\max }}+\frac{\Delta v_{\min }+\Delta v_{\max }}{\Delta v_{i}+\Delta v_{\max }}\right)$

(8) where $\omega=\left(\omega_{1}, \omega_{2}, \ldots, \omega_{n},\right)^{T}$ is the weight vector of $x_{i}(i=1,2, \ldots, n), \omega_{i} \geq 0(i=1,2, \ldots, n)$, and $\sum_{i=1}^{n} \omega_{i}=1$.

When $\omega=(1 / n, 1 / n, \ldots, 1 / n,)^{T}$, Equation (7) takes effect.

The average value of $\rho_{i}$ is taken as the relative origin in the semantic space of users' perceptual needs or in the semantic space of users' typical characteristics, and each correlation coefficient in this semantic space is marked.

\section{Design of Headsets Based on Value Hierarchy Analysis}

\subsection{Analysis of user's characteristics}

The design of 10 headsets were selected in this research, as shown in Figure 3. Based on the analysis of the comprehensive perceptual demands of users on the product, such as and the style and the color (10 headsets were numbered as from left to right, top to bottom), this research aims at determining the product characteristics favored by the target users and carry out a hierarchical analysis of the user's multi-dimensional perceptual demands regarding the product. First of all, the target consumers were determined as young people. This paper defines the characteristics of the user's main personality. The target consumers are expressed as "introvert-outgoing, gentle- fortitude", as shown in Figure 4. 


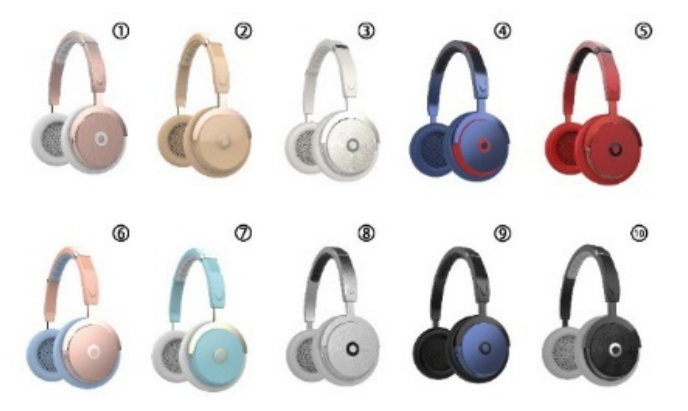

Figure 3.Headsets design samples.

Through experiments and statistical analysis, user's sentimental demands of products were acquired primarily among many perceptual words, which were: 1) $\mathrm{Y}_{1}$-value; 2) $\mathrm{Y}_{2}$ - gender (refers to the style inclining to "masculine" or "feminine"); 3) $\mathrm{Y}_{3}$ - cool; 4) $\mathrm{Y}_{4}$-comfort; 5) $\mathrm{Y}_{5-}$ sportiness; 6) $\mathrm{Y}_{6}$-simple; 7) $\mathrm{Y}_{7}$ - fashion. User's evaluation of 1) outline $G_{1} ; 2$ ) color $G_{2} ; 3$ ) texture $G_{3}$ were selected as indexes of their comprehensive perceptions upon the product, based on which the sentimental demand $Y_{i}=(i=1,2, \ldots, 7)$ was evaluated. In order to simulate actual and accurate situation of design determination, the product development process underwent one year, including 3 parts: user's sentimental demands were acquired through market survey before designing, front-line salesman's sentimental demands were concluded through demand investigation after designing; and after the determination of the final plan, designer's sentimental demands were obtained through survey statistics analysis. The result of the most recent demand test set stronger impact on the final decision, therefore, the weight vector of the test time in these three tests $t_{k}=(k=1,2,3)$ was calculated through $\omega(t)=(1 / 6,2 / 6,3 / 6,)^{T}$. In the experiment, feedback from salespeople made more effect on the final decision. The weight vector of $\mathrm{G}_{1}, \mathrm{G}_{2}, \mathrm{G}_{3}$ were calculated through $w=(0.3,0.4,0.3)^{T}$ according to advices from the evaluation team. Based on this, intuitive fuzzy decisionmaking matrix were established, shown as follows: user's intuitive fuzzy decision-making matrix number about perceptual words Y1, Y2, Y3, Y4, Y5, Y6, Y7 is $\left(D\left(t_{1}\right)\right)$ : $\mathrm{G} 1:(0.5,0.3,0.2)(0.5,0.2,0.2)(0.8,0.1,0.1)(0.7,0.2,0.1)$ $(0.6,0.2,0.2)(0.8,0.1,0.1)(0.7,0.2,0.1) \mathrm{G} 2:(0.6,0.2,0.2)$ $(0.6,0.3,0.1)(0.8,0.1,0.1)(0.6,0.2,0.2)(0.5,0.3,0.2)$

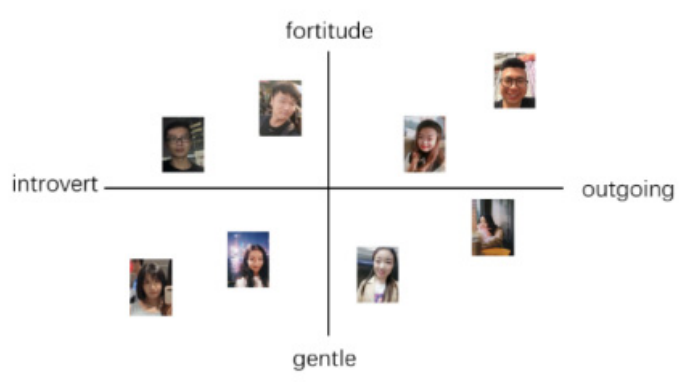

igure 4.User characteristics analysis of headsets.

$(0.7,0.1,0.2)(0.6,0.2,0.2), \mathrm{G} 3:(0.7,0.2,0.1)(0.5,0.4,0.1)$ $(0.6,0.2,0.1)(0.7,0.2,0.1)(0.5,0.3,0.2)(0.5,0.3,0.2)$

$(0.7,0.2,0.1)$, front-line salesman's intuitive fuzzy decision-making matrix number about perceptual words Y1, Y2, Y3, Y4, Y5, Y6, Y7 is $\left(D\left(t_{2}\right)\right): \mathrm{G} 1:(0.8,0.1,0.1)$

$(0.7,0.2,0.1)(0.7,0.2,0.1)(0.7,0.2,0.1)(0.7,0.2,0.1)$

$(0.8,0.1,0.1)(0.5,0.3,0.2), \mathrm{G} 2:(0.4,0.3,0.3)(0.7,0.2,0.1)$

$(0.6,0.3,0.1)(0.5,0.3,0.2)(0.4,0.3,0.3)(0.6,0.2,0.2)$

$(0.8,0.1,0.1), \mathrm{G} 3:(0.6,0.3,0.1)(0.2,0.4,0.4)(0.4,0.4,0.2)$

$(0.8,0.1,0.1) \quad(0.5,0.2,0.3) \quad(0.6,0.2,0.2)$

$(0.4,0.4,0.2)$, and designer's intuitive fuzzy decisionmaking matrix number about perceptual words $\mathrm{Y} 1, \mathrm{Y} 2, \mathrm{Y} 3$, $\mathrm{Y} 4, \mathrm{Y} 5, \mathrm{Y} 6, \mathrm{Y} 7$ is $D\left(t_{3}\right): \mathrm{G} 1:(0.6,0.3,0.1)(0.6,0.3,0.1)$

$(0.7,0.2,0.1)(0.6,0.2,0.2)(0.7,0.2,0.1)(0.8,0.1,0.1)$

$(0.7,0.2,0.1) \mathrm{G} 2:(0.6,0.3,0.1)(0.8,0.1,0.1)(0.6,0.3,0.1)$

$(0.5,0.3,0.2)(0.7,0.2,0.1)(0.6,0.3,0.1)(0.7,0.2,0.1)$ $\mathrm{G} 3:(0.7,0.2,0.1)(0.3,0.6,0.1)(0.5,0.4,0.1)(0.8,0.1,0.1)$

$(0.5,0.4,0.1)(0.4,0.5,0.1)(0.5,0.3,0.2)$.

\subsection{Determination of demand hierarchy}

Step 1: Through DIFWA operator Equation (2), intuitive fuzzy decision-making matrix $D\left(t_{1}\right), D\left(t_{2}\right), \quad D\left(t_{3}\right)$, was integrated as one comprehensive intuitive fuzzy decisionmaking matrix, shown in Table 1.

Step 2: The closeness coefficient of all sementical information was calculated through Equation (6) : $c\left(Y_{1}\right)=0.665, c\left(Y_{2}\right)=0.628, c\left(Y_{3}\right)=0.658, c\left(Y_{4}\right)=0.688$, $c\left(Y_{5}\right)=0.647, c\left(Y_{6}\right)=0.682, c\left(Y_{7}\right)=0.681$

Table 1. Integrated intuitionistic fuzzy decision matrix $D\left(t_{4}\right)$.

\begin{tabular}{cccc}
\hline Perceptual Words & $G_{1}$ & $G_{2}$ & $G_{3}$ \\
\hline$Y_{1}$ & $(0.670,0.208,0.122)$ & $(0.542,0.280,0.177)$ & $(0.670,0.229,0.101)$ \\
$Y_{2}$ & $(0.623,0.262,0.115)$ & $(0.743,0.151,0.106)$ & $(0.308,0.490,0.202)$ \\
$Y_{3}$ & $(0.720,0.178,0.102)$ & $(0.644,0.250,0.107)$ & $(0.488,0.356,0.156)$ \\
$Y_{4}$ & $(0.654,0.200,0.146)$ & $(0.518,0.280,0.201)$ & $(0.786,0.112,0.102)$ \\
$Y_{5}$ & $(0.685,0.200,0.115)$ & $(0.588,0.245,0.167)$ & $(0.500,0.303,0.197)$ \\
$Y_{6}$ & $(0.800,0.100,0.100)$ & $(0.619,0.218,0.163)$ & $(0.492,0.338,0.170)$ \\
$Y_{7}$ & $(0.644,0.229,0.127)$ & $(0.725,0.159,0.116)$ & $(0.512,0.309,0.179)$ \\
\hline
\end{tabular}

Step 3: Based on the closeness coefficient, all plans were ranked as: $Y_{4}>Y_{6}>Y_{7}>Y_{1}>Y_{3}>Y_{5}>Y_{2}$, Hereby, through comprehensive perception evaluation, user's favored sentimental demands were ranked as: $\mathrm{Y}_{4}$-comfort, $\mathrm{Y}_{6^{-}}$ simple, $\mathrm{Y}_{7^{-}}$fashion, $\mathrm{Y}_{1}$-value, $\mathrm{Y}_{3^{-}}$cool, $\mathrm{Y}_{5}$ - sportiness , $\mathrm{Y}_{2^{-}}$ gender. 


\subsection{Semantic correlation analysis of perceptual demand information.}

Users' characteristics to stand-riding scooters were defined as the user set "introvert-outgoing, gentlefortitude ". Among all pairs of semantic words, higher score stands for fortitude or outgoing users while lower score stands for gentle or introvert. Perceptual demands of users were defined as the characteristic set, i.e. "value , gender , cool, comfort, sportiness, simple, fashion". Through investigation to users, their inclinations of abovementioned sentimental information and sets were collected. The accuracy of data describing user's perceptual demand will increase with the rising of user number.

Table 2. Perception correlation data.

\begin{tabular}{c|c|c|c|c|c|c|c}
\hline Perceptual Words & Value & Gender & Cool & Comfort & Sportiness & Simple & Fashion \\
\hline $\begin{array}{c}\text { (Fortitude, } \\
\text { Gentle })\end{array}$ & $(0.6,0.3)$ & $(0.5,0.3)$ & $(0.7,0.2)$ & $(0.7,0.3)$ & $(0.6,0.3)$ & $(0.6,0.3)$ & $(0.6,0.3)$ \\
\hline $\begin{array}{c}\text { (Outgoing, } \\
\text { Introvert) }\end{array}$ & $(0.6,0.3)$ & $(0.5,0.3)$ & $(0.7,0.2)$ & $(0.6,0.3)$ & $(0.5,0.3)$ & $(0.6,0.3)$ & $(0.6,0.3)$ \\
\hline
\end{tabular}

The perception correlation data of user sets and sentimental demand information are given in Table 2, every pair of information among which was described by indexes $(\mu, v)$, where $\mu$ refers to membership degree and $v$ to non-membership degree. All product plans are defined as set $\mathrm{P}, P=\left\{P_{1}, P_{2}, \ldots, P_{10}\right\}$, and perception characteristics of all plans are listed in Table 3 . According

Table 3. Product perception characteristics data.

\begin{tabular}{cccccc}
\hline P & $P_{1}$ & $P_{2}$ & $P_{3}$ & $P_{4}$ & $P_{5}$ \\
\hline Y1 & $(0.6,0.4)$ & $(0.6,0.4)$ & $(0.6,0.3)$ & $(0.5,0.4)$ & $(0.5,0.5)$ \\
Y2 & $(0.6,0.3)$ & $(0.5,0.5)$ & $(0.4,0.6)$ & $(0.6,0.4)$ & $(0.6,0.3)$ \\
Y3 & $(0.5,0.4)$ & $(0.4,0.5)$ & $(0.4,0.5)$ & $(0.6,0.4)$ & $(0.5,0.4)$ \\
Y4 & $(0.6,0.4)$ & $(0.6,0.4)$ & $(0.5,0.5)$ & $(0.5,0.5)$ & $(0.5,0.5)$ \\
Y5 & $(0.5,0.5)$ & $(0.4,0.5)$ & $(0.6,0.4)$ & $(0.6,0.3)$ & $(0.6,0.4)$ \\
Y6 & $(0.5,0.5)$ & $(0.6,0.3)$ & $(0.7,0.2)$ & $(0.5,0.5)$ & $(0.5,0.5)$ \\
Y7 & $(0.6,0.3)$ & $(0.5,0.5)$ & $(0.4,0.5)$ & $(0.5,0.5)$ & $(0.6,0.4)$ \\
\hline P & $P_{6}$ & $P_{7}$ & $P_{8}$ & $P_{9}$ & $P_{10}$ \\
\hline Y1 & $(0.5,0.4)$ & $(0.6,0.3)$ & $(0.5,0.4)$ & $(0.6,0.3)$ & $(0.5,0.4)$ \\
Y2 & $(0.7,0.2)$ & $(0.5,0.5)$ & $(0.4,0.5)$ & $(0.7,0.2)$ & $(0.6,0.3)$ \\
Y3 & $(0.5,0.5)$ & $(0.4,0.5)$ & $(0.5,0.4)$ & $(0.6,0.3)$ & $(0.5,0.5)$ \\
Y4 & $(0.5,0.4)$ & $(0.6,0.4)$ & $(0.4,0.6)$ & $(0.5,0.4)$ & $(0.5,0.4)$ \\
Y5 & $(0.5,0.4)$ & $(0.5,0.5)$ & $(0.4,0.6)$ & $(0.7,0.3)$ & $(0.6,0.3)$ \\
Y6 & $(0.5,0.4)$ & $(0.6,0.4)$ & $(0.6,0.4)$ & $(0.5,0.5)$ & $(0.5,0.4)$ \\
Y7 & $(0.5,0.4)$ & $(0.5,0.4)$ & $(0.5,0.4)$ & $(0.6,0.3)$ & $(0.6,0.4)$ \\
\hline
\end{tabular}

Diagnosis was carried out to $P_{i}=(1,2,3,4,5,6,7,8,9,10)$ by the correlation measure Equation (1)and the correlation

weight $\left(Y_{i}, \omega_{i}\right)$ between sentimental demand and user characteristics the results shown in Table 4 in detail.

to the closeness coefficient $c\left(Y_{i}\right)$, the correlation weight $\left(Y_{i}, \omega_{i}\right)$ between sentimental information and user characteristics respectively defined as: $\left(\mathrm{Y}_{4}, 0.3\right),\left(\mathrm{Y}_{6}, 0.2\right)$, $\left(\mathrm{Y}_{7}, 0.1\right),\left(\mathrm{Y}_{1}, 0.1\right),\left(\mathrm{Y}_{3}, 0.1\right),\left(\mathrm{Y}_{5}, 0.1\right),\left(\mathrm{Y}_{2}, 0.1\right)$. Table 4. Integrated perception correlation data of products oriented by the user characteristics.

\begin{tabular}{c|c|c|c|c|c|c|c|c|c|c}
\hline Plans & $\mathrm{P}_{1}$ & $\mathrm{P}_{2}$ & $\mathrm{P}_{3}$ & $\mathrm{P}_{4}$ & $\mathrm{P}_{5}$ & $\mathrm{P}_{6}$ & $\mathrm{P}_{7}$ & $\mathrm{P}_{8}$ & $\mathrm{P}_{9}$ & $\mathrm{P}_{10}$ \\
\hline Outgoing & 0.773 & 0.767 & 0.680 & 0.871 & 0.625 & 0.775 & 0.900 & 0.917 & 0.813 & 0.775 \\
\hline Fortitude & 0.833 & 0.683 & 0.792 & 0.742 & 0.658 & 0.689 & 0.767 & 0.675 & 0.750 & 0.650 \\
\hline
\end{tabular}

The average "outgoing correlation value" and "fortitude correlation value" of 10 product plans $(0.791$, $0.724)$ were selected as the "relative origin" in the semantic space "outgoing, fortitude" of the target user group correspondingly. And the position of the comprehensive perception of "value, gender , cool, comfort, sportiness, simple, fashion." in the semantic space of target user group can thus be acquired, as shown in Figure 5. 


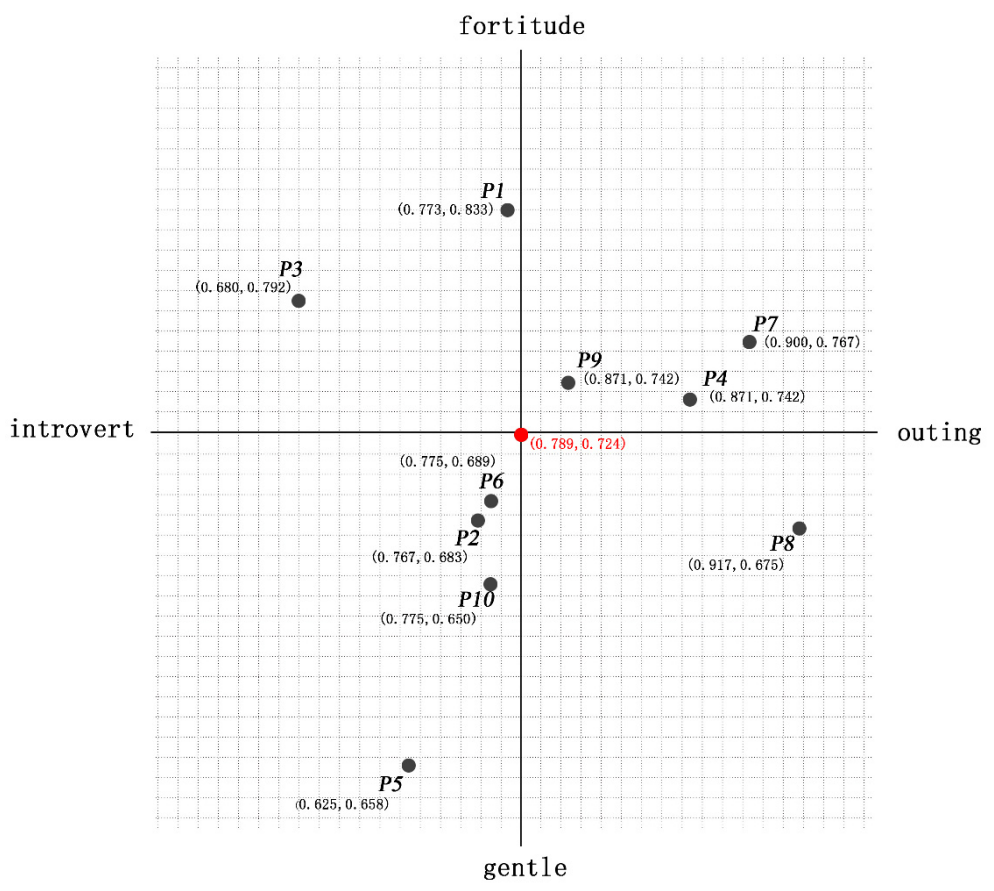

Figure 5. User characteristics analysis of headsets.

\section{Conclusion}

In terms of the users' intricate perceptual demands when they are facing products, the study adopted intuitionistic fuzzy multi-attribute decision-making approach to rank the perceptual demand of users and find " $M R_{j}$ ", the emotional semantic axis. The adoption of correlation measure of intuitionistic fuzzy set to calculate the hierarchy relevance between perceptual demand semantic set and emotional semantic axis may effectively conduct hierarchy correlation clustering on perceptual information. The example verifies that the correlation calculation measure used in this paper can comprehensively sense the ideal product's "position" in the semantic space of typical user group. What's more, it expressed the hierarchy of demands of users more clearly and intuitively, and improved the scientificity in product design. This method can process human emotion towards object from a comprehensive perspective and can be applied to human dynamic emotional semantic correlation and clustering analysis beyond product design evaluation and decisionmaking.

In further studies, the method proposed in this paper can be used to work out more precise decision for product plan with multi-perception and multi-attribute evaluation group based on more data of user information.

\section{Acknowledgements}

This research was financially supported by the National Social Science Fund of China(Grant No.18BG132)

\section{References}

1. Zhang Jf, Jin XT.(2019) Sensitivity ElementOriented Product Image Design: Taking Smart Speaker as an Example.Design Research, 9: 115-120.

2. Wang C, Zhao W, Wang J, et al.(2016) An Ontology Based Multi-Dimensional User Requirement Acquisition. Computer Integrated Manufacturing Systems, 22: 908-916.

3. HuiMJ,C.K. Kwong,G.E. Okudan Kremer;;W.-Y. Park(2019) Dynamic modelling of customer preferences for product design using DENFIS and opinion mining. Elsevier Ltd, 42

4. Li SB, Quan HF, Hu JJ, Wu YM, Zhang AS(2018) Perceptual evaluation method of products based on online reviews data driven. Computer Integrated Manufacturing Systems, 24: 752-762

5. Wang Y. (2016) Product Color Design. Chemical Industry Press, Beijing.

6. $\mathrm{Xu} \mathrm{ZS.} \mathrm{(2008)} \mathrm{Theroy} \mathrm{and} \mathrm{Application} \mathrm{of}$ Intuitionistic fuzzy Sets. Science Press, Beijing.

7. Cheng XF, Zhu J, Zhou EM.(2017) Research of the customer requirement model for product family based on conjoint analysis and fuzzy clustering. Chinese Journal of Engineering Design, 24: 8-17+26.

8. Yao X, Hu HY, Li JY.(2016) Research on matching of user's emotional demands level and product design feature. Wuhan University of Technology(Social Science Edition), 29: 304-307.

9. Cao GZ, Shi K Wang T.(2019) User-Requirement Analysis Method Based on Multi-Dimensional Sensibility. Packaging Engineering, 40: 119-127. 